\title{
Die meistzitierten Arbeiten des 41. Jahrgangs der Psychiatrischen Praxis - Herzlichen Glückwunsch!
}

Autoren

Institut
Philipp Reimann, Steffi G. Riedel-Heller

Institut für Sozialmedizin, Arbeitsmedizin und Public Health (ISAP), Medizinische Fakultät, Universität Leipzig

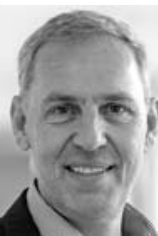

Martin

Lambert

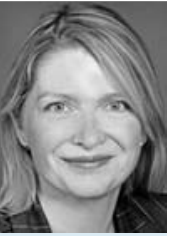

Uta Gühne
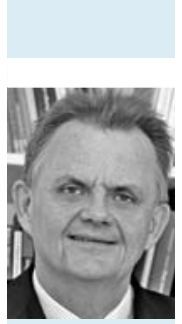

Günther

Wienberg

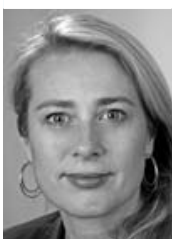

Anne

Karow

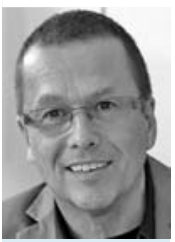

Ingmar Steinhart

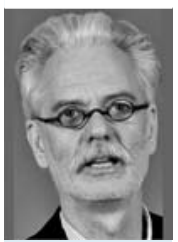

Holger Hoffmann
Bibliografie

DoI http://dx.doi.org/ 10.1055/s-0042-122926

Psychiat Prax 2017; 44: 6

(c) Georg Thieme Verlag KG Stuttgart - New York ISSN 0303-4259

Korrespondenzadresse Prof. Dr. med.

Steffi G. Riedel-Heller, MPH Direktorin, Institut für

Sozialmedizin, Arbeitsmedizin und Public Health (ISAP),

Universität Leipzig,

Medizinische Fakultät

Philipp-Rosenthal-Straße 55

04103 Leipzig

Steffi.Riedel-Heller@medizin.

uni-leipzig.de

\section{Integrierte Versorgung - Hamburger Modell}

Als meistzitierte Originalarbeit küren wir eine zweiteilige Publikation von Prof. Dr. med. Martin Lambert und Prof. Dr. med. Anne Karow, beide aus der Klinik für Psychiatrie des Universitätsklinikums Hamburg-Eppendorf. Sie stellen das „Hamburger Modell“, ein integriertes Versorgungsmodell für Psychosekranke nach §140 SGB $\mathrm{V}$ vor und berichten eindrucksvolle Ergebnisse nach 2 und 4 Jahren [1, 2]. Das sektorenübergreifende Behandlungsmodell umfasst ein therapeutisches Assertive Community Treatment (ACT) sowie alle voll- und teilstationären und ambulanten Behandlungen.

\section{Psychotherapie der Depression im Alter} $\nabla$

Die meistzitierte Übersichtsarbeit stammt aus der Feder von Dr. rer. med. Uta Gühne vom Institut für Sozialmedizin, Arbeitsmedizin und Public Health (ISAP) der Universität Leipzig. Die Übersicht widmet sich der Wirksamkeit von Psychotherapie bei älteren depressiven Menschen [3]. Die Recherche ergibt: Psychotherapeutische Interventionen zur Behandlung depressiver Störungen im höheren Lebensalter sind effektiv, aber es gibt noch deutlichen Forschungsbedarf, insbesondere zur Wirksamkeit von Psychotherapie bei hochaltrigen Patientinnen und Patienten mit kognitiven und sensorischen Beeinträchtigungen sowie komorbiden körperlichen Erkrankungen.

\section{Funktionales Basismodell gemeinde- psychiatrischer Versorgung \\ $\nabla$}

Das meistzitierte Editorial legen Prof. Dr. Ingmar Steinhart, Leiter des Instituts für Sozialpsychiatrie Mecklenburg-Vorpommern der Ernst-Moritz Arndt-Universität Greifswald, und Prof. Dr. Günther Wienberg von der Hochschule Fulda vor. Ihr funktionales Basismodell hat Furore gemacht [4] und wurde jüngst erweitert [5].

\section{Berufliche Rehabilitation: First Place, then Train \\ $\nabla$}

Erst platzieren, dann trainieren - so sollte berufliche Rehabilitation für schwer psychisch Kranke erfolgen. Daran lässt PD Dr. med. Holger Hoffmann von den Universitären Psychiatrischen Diensten Bern in seinem meistzitierten Debattenbeitrag keinen Zweifel [6]. Die Evidenz spricht Bände. Diese Debatte hat weitere Beiträge inspiriert $[7,8]$.

Wir gratulieren den Autorinnen und Autoren ganz herzlich!

\section{Literatur}

1 Lambert M, Bock T, Daubmann A et al. Integrierte Versorgung von Patienten mit psychotischen Erkrankungen nach dem Hamburger Modell: Teil 1. Rationalen, Behandlungsmodell und Ergebnisse der Vorstudie. Psychiat Prax 2014; 41: 266-273

2 Karow A, Bock T, Daubmann A et al. Integrierte Versorgung von Patienten mit psychotischen Erkrankungen nach dem Hamburger Modell: Teil 2. Ergebnisse des 2- und 4-Jahres-Langzeitverlaufs. Psychiat Prax 2014; 41: 527-265

3 Gühne U, Luppa M, König H-H et al. Ist Psychotherapie bei depressiven Erkrankungen im Alter wirksam? Ein systematischer Überblick. Psychiat Prax 2014; 41: $415-432$

4 Steinhart I, Wienberg G. Plädoyer für ein funktionales Basismodell gemeindepsychiatrischer Versorgung? Psychiat Prax 2014; 41: 179-181

5 Steinhart I, Wienberg G. Das Funktionale Basismodell für die gemeindepsychiatrische Versorgung schwer psychisch kranker Menschen - Mindeststandard für Behandlung und Teilhabe. Psychiat Prax 2016; 43: $65-68$

6 Hoffmann H. Berufliche Rehabilitation. First Place, then Train - Pro. Psychiat Prax 2014; 41: 293-294

7 Kilian H. Supported Employment - ein falsches Konzept für Deutschland? - Pro. Psychiat Prax 2016; 43: $242-243$

8 Stengler K, Becker T. Supported Employment - ein falsches Konzept für Deutschland? - Kontra. Psychiat Prax 2016; 43: 243-244 